\title{
MSA SYSTEM CONCEPT FOR MEDITERRANEAN SEA SURVEILLANCE
}

\author{
Fabrizio BERIZZI, Amerigo CAPRIA, \\ Enzo DALLE MESE, Amleto GABELLONE, \\ Marco MARTORELLA and Walter MATTA
}

\begin{abstract}
This paper focuses on general considerations and ideas on a Maritime Situational Awareness (MSA) system concept addressing specifically the surveillance of the Mediterranean Sea. It presents analysis of the main potentials and limits of existing Maritime Surveillance Systems (MSSs) operating on the Mediterranean Sea and identified technology gaps. The authors then propose MSA system architecture, applications, requirements and sensors. The paper concludes with a few considerations on signal processing and data fusion techniques, required for the implementation of the MSA system capabilities.
\end{abstract}

Keywords: Maritime Security, Maritime Situational Awareness, OTH-RADARs, ISAR Images.

\section{Introduction}

Mediterranean sea surveillance capability is becoming a crucial requirement in order to deal with national and international safety issues such as clandestine immigration, naval traffic control, illegal actions surveillance (e.g. oil spills, building abusiveness, chemical pollution, etc.) as well as other potential threats ranging from piracy and organised crime to full-scale naval warfare. A secure maritime domain requires enhanced transparency and information sharing to ensure situational awareness and to enable an adequate operational response from the relevant national and international, military and civil agencies.

A key requirement to have Maritime Situational Awareness (MSA) is to collect situational data and produce and distribute a unified presentation of the maritime situation, the MSA Picture. Existing Maritime Surveillance Systems (MSSs) of different Mediterranean European or other countries often operate in a limited sea area mainly including own coasts without sharing collected information to other MSSs to produce 
dedicated picture (CROP - Common Reduced Operational Picture) for particular users. Moreover, such systems are often designed to specific operational scope, like location of cooperating naval units, and they are not fully able to face current threats like detection of vessels involved in terrorist or illegal activities. Existing MSSs show a number of shortfalls, including:

- Limited information availability on non-military and non-cooperating shipping;

- Lack of system-to-system integration and appropriate interfacing;

- Limited capability to fuse a wide range of information and produce a full unified MSA Picture;

- Limited capability to exchange data between nations and agencies;

- Limited spatial and temporal sensory coverage (e.g. limited geographical area coverage: individual sensors and indeed systems each provide coverage of limited areas);

- Limited availability of information on vessel identity and activity;

- Susceptibility to error and deception in data supplied, e.g. 'spoofing' of AIS (Automatic Identification System) data;

- Lack of a multi-level security framework in systems to enable integration of agencies with differing data security level requirements.

The goal of this paper is to analyse and discuss the main aspects of an MSA system, mainly devoted to the surveillance of the Mediterranean Sea. The system concept will be defined, starting from a critical analysis of deficits of the existing system and proposing the MSA architecture, applications and requirements. Advanced sensors that could be potentially included on the surveillance system and not currently fully exploited will be addressed. The paper concludes with an excursus of a few innovative signal processing and fusion techniques that could enhance the performance of existing MSSs, to be implemented in the MSA system.

\section{MSA System Concept}

\section{Potential and limits of current maritime surveillance systems}

Maritime Situational Awareness at EU level and specifically for Mediterranean surveillance is carried out by national authorities to detect, identify and counter illegal activities and/or potential security and safety threats. Other domains, such as fisheries and environmental protection, shipping safety and immigrants control show limits in MSA concepts because surveillance is based on laws and regulations that govern the above domains. 
A basic taxonomy for MSA focuses on cooperative and non-cooperative systems: the former include reporting/messaging systems such as VMS (Vessel Monitoring System), AIS and many other non-automatic linking systems which rely on the ships to provide information. The latter include sensor systems, such as radars, satellites and cameras that collect traffic and navigation information without ships' cooperation.

Another distinction is between intentional and non-intentional infringements that an MSA system is supposed to identify and solve. In the first case a non-cooperative MSA system is essential for correct threat classification, as any cooperative information could be fake and misleading. Cooperative MSA systems are in any case necessary and complementary to non-cooperative ones in order to manage the huge amount of compliant information.

When an a potential threat or an illegal activity has been found, a MSS needs a sort of dual use system: used as a forward observation and communication system in order to allow a close-up inspection, gather additional information and identify the infringement; and used as a countermeasure system in order to intercept and/or counter the threat. Depending on scenarios, naval, aerial or land means are deployed for the above purposes. These means or assets could be an integral part of the whole surveillance system.

According the particular scenario and their needs, the Mediterranean countries have made various choices about the requirements, performances and deployments of the MSS. This leads to disparity of different MSS used and the intrinsic limitations of each system do not allow a complete MSA picture, e.g. the VMS system with its 2hour reporting, the VTS radars that only detect large ships, etc. Even if each system successfully covers different subsets of the maritime traffic according its technical specifications and requirements, no single system allows a complete overview of all vessels within a specific area. These limits can be overcome through the integration of the information gathered form different surveillance systems in use by the government authorities of Mediterranean countries. This integration concept is valid not only for cooperative systems, such as VMS and AIS, but also for non-cooperative ones.

In non-cooperative systems sensor performance is generally limited by environmental conditions and target dimensions and response to the sensor's pulses. This is especially true for small boats that could be used for illegal immigration and terrorism. Data integration of different kinds of sensors covering a certain area would allow a very low false alarm rate. A drawback of a complete MSA picture is the availability of a great amount of targets with the consequence of an incorrect identification of a potential threat. Therefore combining data coming from cooperative systems allows 
filtering of known and 'friendly' vessels, leaving fewer unknown ships or 'foes' in the picture and reducing the amount of potential risk targets that need a closer attention.

Sometimes not merging data from different systems could be a deliberate choice, driven by probably legitimate requirements based on cost/benefit and efficiency considerations (i.e., the Spanish SIVE - a system used to detect small vessels is not coupled with the VTS system which covers part of the same area, probably because much more sensitive traffic pictures coming from SIVE give no useful info to VTS operators and vice versa). Therefore, ad hoc integration architectures have to be developed to automatically manage a more congested MSA picture and allow operators making best use of all the available information.

Finally, national security issues could pose an additional limitation to the integration of MSS data for commercial, political or military reasons. Integration of different security regulated surveillance systems has to be performed when possible, together with solutions for sharing information and managing unclassified information extracted from classified systems.

\section{Architecture}

To timely respond to Mediterranean maritime threats and to improve the quality of the results, the MSA system should consists of a federation of existing assets/systems and the integration of new systems in order to build a 'system of systems' able to establish a common Recognised Maritime Picture (RMP) across the various operations centres of Mediterranean nations.

An architecture for the MSA system should enhance existing systems by providing a level of "inter-system federation \& integration" via the implementation of a technical infrastructure for information exchange and processing. Existing systems will also require enhancement in order to meet the challenges arising from the potential threats in the current and future environments.

A possible preliminary architecture of the MSA is depicted in Figure 1. The nodes of the MSA network are the national Maritime Surveillance Centres (MSCs) whose main task is to integrate and process all information coming from Maritime Surveillance Systems (MSSs). The main task of the MSS is to collect all data coming from sensors or other sub-systems relevant to a given sea area, fuse data to enhance ship detection and identification, produce maritime situational data synchronized in time and space. Several sub-systems can be involved such as Automatic Identification System (AIS), Maritime Air Assets, Maritime Vessels, Shore-Based Assets, Spacebased Maritime Surveillance Systems (SMSS), including Earth Observation, Reconnaissance and Surveillance Satellite Systems, Integrated Communication (COM) and 
data link infrastructure, Command, Control, Communication, Computer, Intelligence (C4I) systems, etc.

The MSS also controls sensor and sub-system functionalities in accordance with specific operational tasks. As an example, the system can switch from surveillance modality to tracking functionality once a set of targets of interest have been selected.

MSCs are connected to the MSSs and collect all data coming from them. A further data processing is performed to create CROP or RMP depending on user requirements. MSA pictures are exchanged and shared to other National MSC according to suitable operational strategies. If at least one MSC per Mediterranean country is available, the MSA system will become a unified centre for Mediterranean situation awareness.

\section{MARITIME SITUATIONAL AWARENESS (MSA) NETWORK SYSTEM}

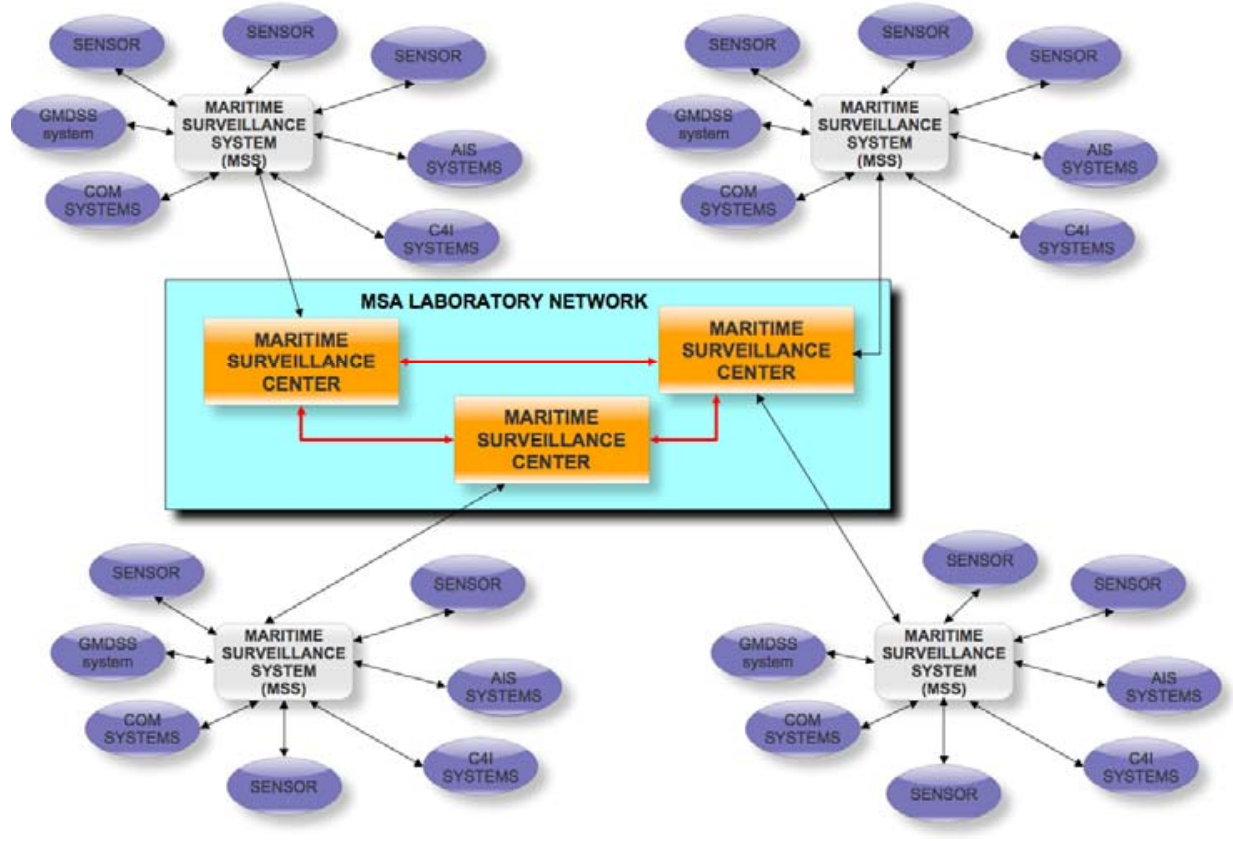

Figure 1: MSA Network System. 


\section{Applications}

MSA system should be applicable to both civil and military scenarios, including:

- High seas surveillance;

- Coastal surveillance;

- Maritime border control;

- Prevention of illegal immigration;

- Effective handling of emergencies and disasters;

- Coordination of Search and Rescue (SAR).

Different types of sensor data analysis can be considered:

- $\quad$ ship traffic monitoring;

- detection and classification of non-cooperative vessels;

- identification of suspected targets;

- oil spill detection and identification of responsible ship;

- sea current mapping;

- $\quad$ ship routing;

- detection of 'abnormal changes,' i.e., detection of abnormal events across and within large arrays of 'normal events';

- increasing the overall spatial coverage of the sensing systems;

- integration of space-based surveillance data.

\section{Requirements}

All major Mediterranean countries are more or less cooperating on issues concerning illegal immigrant control, fisheries and shipping safety. But achieving a complete MSA picture requires an effective MSA "system of systems" concept where each MSA node will take advantage from the others even controlling confined area sectors or using different sensors. In order to realize this idea, all Mediterranean governments should agree on a feasible MSA integration roadmap based on:

1. Existing exchanges among the different authorities across borders and across sectors;

2. Updated operational requirements;

3. Legal, technical and administrative requirements. 
Requirements of points 1 and 2 are easily drawn through the analysis of suggested MSA architecture and applications. The requirements of point 3 warrant a further consideration.

Legal requirements need to overcome the limitations already outlined above. First of all, a MSA system requires that all the information provided by different sources and for different purposes needs to be linked, exchanged and integrated. This means that data exchange has to take into account existing obligations and regulations among Mediterranean states. Another issue is information security of classified data. Confidentiality implies that data may not be passed to third parties and can be regulated by ad hoc non disclosure agreements. The VMS regulations, the VTMIS (Vessel Traffic Management and Information System) directive, the SOLAS (Safety Of Life At Sea) convention are some of the current regulations managing the exchange and integration of MSS data. Further legal issues relate to the protection of personal data, datasharing policy and re-use of public sector information. Another requirement to consider is the restriction imposed by the software architecture and licensing.

Technical requirements can be drawn by use of existing initiatives and outcomes of research projects. EUROSUR (European Border Surveillance System) is an important programme to test the technical aspects of integrated maritime surveillance. The system technology showed in this paper is fully addressed by EUROSUR requirements which are based on a "system of existing systems" built in three different phases:

- Upgrading and extending national border surveillance systems and interlinking national infrastructures in a communication network for border control authorities;

- Improvement of performance of other surveillance tools (e.g. satellites) and the creation of a pre-frontier intelligence picture;

- Creation of a common information sharing environment for the EU maritime domain.

Interesting MSA requirements stem from LIMES and MARISS projects. They demonstrate the added value of earth observations (both optical and radar) from space, combined with other technologies for monitoring shipping. Information provided by cooperative on-board systems (e.g. AIS, VMS) are merged with information coming from satellite images in order to identify potential infringements.

Administrative requirements are focal points of a MSA system as the administrative set-up at national level is different and sometimes difficult to be fused to others. The BORTEC study shows over 50 authorities dealing with maritime and border surveillance in the seven EU Mediterranean Countries (Greece, France, Italy, Cyprus, Malta, Spain, Slovenia). The case study of Italy is very significant (see table 1). 
Therefore an integrated maritime surveillance policy has not to duplicate or set up a new administrative model but needs to be based on coordination and cooperation among all existing administrative structures through a system of system architecture and exploiting all the advanced technology in communication systems and sensors.

\section{Advanced sensors}

A wide range of sensors and other data sources can be included to be part of the MSA system. Some of them are:

- Radar, including Synthetic Aperture Radar (SAR), Moving Target Indication (MTI), Inverse Synthetic Aperture Radar (ISAR), OTH Radar;

- Electro-Optical (EO) Sensors;

- Electronic Support Measures (ESM);

- Sonars;

- Automatic Identification System (AIS);

- Own data reports;

- Observations;

Table 1. Italian authorities dealing with maritime and border surveillance (BORTEC study).

\begin{tabular}{ll}
\hline Authority & Responsibility \\
\hline Guardia di Finanza & $\begin{array}{l}\text { Coordinating activities of all national means patrolling } \\
\text { against illegal immigration }<=24 \mathrm{~nm} \text { off coast }\end{array}$ \\
\hline Navy & $\begin{array}{l}\text { Coordinating activities of all national means patrolling } \\
\text { against illegal immigration }>24 \mathrm{~nm} \text { off coast (international } \\
\text { waters) }\end{array}$ \\
\hline Coast Guard & Coordinating SAR at sea \\
\hline Min. Interior - Immigration & $\begin{array}{l}\text { Illegal immigration over sea - overall coordination of all } \\
\text { activities and analysis of all information from the other au- } \\
\text { thorities. }\end{array}$ \\
\hline $\begin{array}{l}\text { Min. Interior - Prefect (local } \\
\text { authority) }\end{array}$ & $\begin{array}{l}\text { Coordination of regional patrol plans (incl. continuity land - } \\
\text { sea) }\end{array}$ \\
\hline
\end{tabular}




\begin{tabular}{|c|c|c|c|}
\hline System & Owner authority & Purpose & Cross-links \\
\hline \multirow[t]{3}{*}{ C4I } & $\begin{array}{l}\text { Guardia di } \\
\text { Finanza }\end{array}$ & $\begin{array}{l}\text { Coordinate the surveillance activi- } \\
\text { ties of the Guardia di Finanza at } \\
\text { sea, which are aimed at combating } \\
\text { illegal activities. }\end{array}$ & \\
\hline & & $\begin{array}{l}\text { Strategic management (planning } \\
\text { etc.). Tactical C\&C. }\end{array}$ & \\
\hline & & Assignment of $\mathrm{C} \& \mathrm{C}$ to local OC. & \\
\hline $\begin{array}{l}\text { Coastal radar } \\
\text { GF* }\end{array}$ & $\begin{array}{l}\text { Guardia di } \\
\text { Finanza * }\end{array}$ & $\begin{array}{l}\text { Integrated advanced police system } \\
\text { for coastal surveillance } *\end{array}$ & \\
\hline \multirow[t]{2}{*}{ MCCIS } & Navy & C4I for maritime assets & \\
\hline & & $\begin{array}{l}\text { Used also for control of illegal } \\
\text { immigration }\end{array}$ & \\
\hline $\begin{array}{l}\text { Coastal radar } \\
\text { Navy }\end{array}$ & Navy & & $\begin{array}{l}\text { Future: connect Coastal Sur- } \\
\text { veillance OC with other CAs. } \\
*\end{array}$ \\
\hline \multirow[t]{2}{*}{ V-RMTC } & Navy & $\begin{array}{l}\text { Exchange unclassified info on mer- } \\
\text { chant vessel traffic }\end{array}$ & $\begin{array}{l}\text { Integrates vessel traffic data } \\
\text { (AIS) of various countries }\end{array}$ \\
\hline & & $\begin{array}{l}\text { Enhance Med Sea Navies coopera- } \\
\text { tion }\end{array}$ & \\
\hline \multirow[t]{4}{*}{ VTMIS } & Coast Guard & Enhancing safety and efficiency of & Interoperable with SSN \\
\hline & & maritime traffic, improve emer- & Web access \\
\hline & & gency response & $\begin{array}{l}\text { Integration with SAR / } \\
\text { GMDSS }\end{array}$ \\
\hline & & & $\begin{array}{l}\text { Future objective: support co- } \\
\text { ordinated actions of various } \\
\text { CAs }\end{array}$ \\
\hline
\end{tabular}

\begin{tabular}{llll}
\hline ARES & \multicolumn{3}{c}{$\begin{array}{l}\text { Automated reporting system for } \\
\text { Italian-flag merchant vessels }>1600 \\
\text { GT anywhere in the world }\end{array}$} \\
\hline ADRIREP & & $\begin{array}{l}\text { Adriatic Sea International Report- } \\
\text { ing System }\end{array}$ & Feeds into VTMIS \\
\hline SSAS & Ship Security Alert System & \\
\hline VMS & Coast Guard & & $\begin{array}{l}\text { Integration of VMS and } \\
\text { VTMIS allows CG to find } \\
\text { fishing vessels w/o blue box. }\end{array}$ \\
\hline $\begin{array}{l}\text { New inte- } \\
\text { grating sys- } \\
\text { tem of Min. }\end{array}$ & $\begin{array}{l}\text { Min. Interior }- \\
\text { Immigration } \\
\text { Interior } *\end{array}$ & $\begin{array}{l}\text { Integrate data from all surveillance } \\
\text { systems, for combating illegal im- } \\
\text { lice Directorate } \\
\text { migration * }\end{array}$ & Combines various systems * \\
\hline Patrol means & $\begin{array}{l}\text { Guardia di } \\
\text { Finanza }\end{array}$ & $\begin{array}{l}\text { Surveillance of major routes from } \\
\text { High Seas to Territorial waters } \\
\text { Surveillance of Territorial waters, } \\
\text { sometimes out in Contiguous zone }\end{array}$ & \\
& & $\begin{array}{l}\text { Navy's means under CINCNAV } \\
\text { have combating illegal immigration } \\
\text { as secondary mission }\end{array}$ & \\
\hline Patrol means & Navy & &
\end{tabular}


- Other sources, including vehicle movement plans and databases, government and non-government non-military agencies, commercial sources, public domain, open source, Internet.

Among all in the list above, there are some advanced sensors that deserve a specific focus because they could improve the overall performance of the MSA system in terms of spatial and time coverage, detection, tracking and classification. The following sub-sections will take into analysis ISAR systems, OTH radar, satellite constellations and Unmanned Aerial Vehicle (UAV) sensors.

\section{ISAR systems}

Inverse Synthetic Aperture Radar (ISAR) is a well-consolidated technique for imaging targets. High bandwidth signals are exploited to get high resolution along range direction through signal compression in the fast time domain. High cross-range resolution can be achieved by coherently processing echoes coming from different target aspect angles. Spatial resolution up to the magnitude order of a few centimetres can be obtained. Ultrahigh resolution images allows target details to be discovered with a consequent benefits in term of classification. References $1-10$ present recent advances in this topic.

Examples of ship and airplane ISAR images are presented in Figure 2. It is worth noting that ship main mast and cranes can be easily recognised in the images, as well as airplane nose, fuse and wings. Information extractable from an ISAR image can be also improved if multiple polarization radar were available. Four ISAR polarimetric images of a non-cooperating ship are presented in Figure 3. It is quite easy to see that polarization diversity allows different details of the targets to be enhanced. The whole polarimetric signal can be also processed globally to get a polarimetric ISAR image, as plotted in Figure 4 coded in RGB. Polarization responses of target scatterers provide information on their shape like dihedral, trihedral, plane, helix an others, thus improving the classification and identification process.

The ISAR kit can be mounted on a coherent radar carried out on different platforms, either stationary or air or space-based. Therefore, all coherent radars belonging to an existing surveillance system can be theoretically equipped with an ISAR processor. ISAR seems to be a promising technique for improving the overall performance of the MSA system and it could be a valuable upgrade of existing surveillance coherent radars.

\section{OTH-R Systems}

A time-space continuous observation of the Mediterranean Sea requires a very wide area surveillance capability. A possible response to this critical requirement is represented by HF sky-wave (ionosphere reflection) Over The Horizon (OTH) radar. ${ }^{13-20}$ 

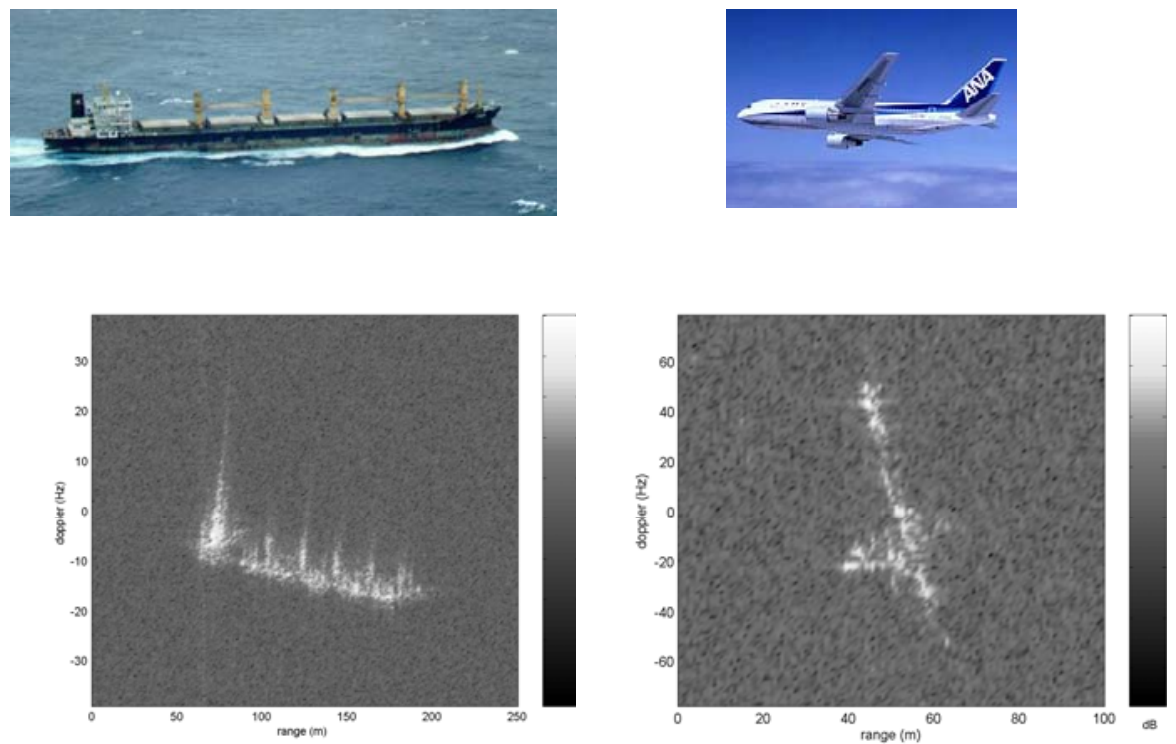

a)

b)

Figure 2: ISAR images of a) a bulk loader and b) Boeing 737.

Distances well beyond the horizon can be reached by exploiting the effect of ionosphere reflection, since EM waves in the HF band are gradually bended through the ionosphere. The ionosphere acts like an electromagnetic mirror at these frequencies. The signal is reflected back by the Earth to the radar system that can perform the detection operations.

This type of radar achieves the remarkable advantage of a very wide and time continuous coverage that ranges from $600 \mathrm{~km}$ up to $3000 \mathrm{~km}$. The cost-effectiveness of such a system is outstanding if compared with costs of possible alternatives to HFOTH radar such as satellite constellations, fleet of airplane flying over the area under surveillance, or a set of radars located along the Mediterranean coast.

The use of low frequencies implies low spatial resolution at the order of a few kilometres. Therefore, such a system can be considered as a theatre early-warning surveillance radar that must be complemented by other high resolution systems for target identification. 

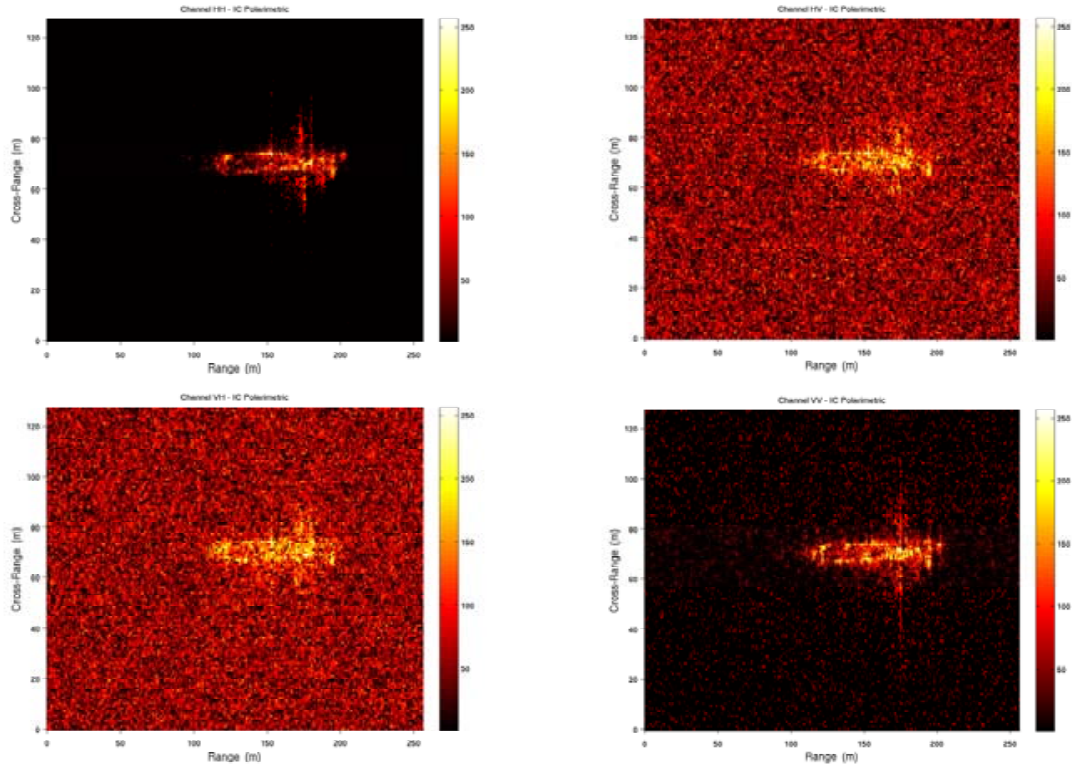

Figure 3: Four channels polarimetric ISAR images.

RGB Image - IC Polarimetric
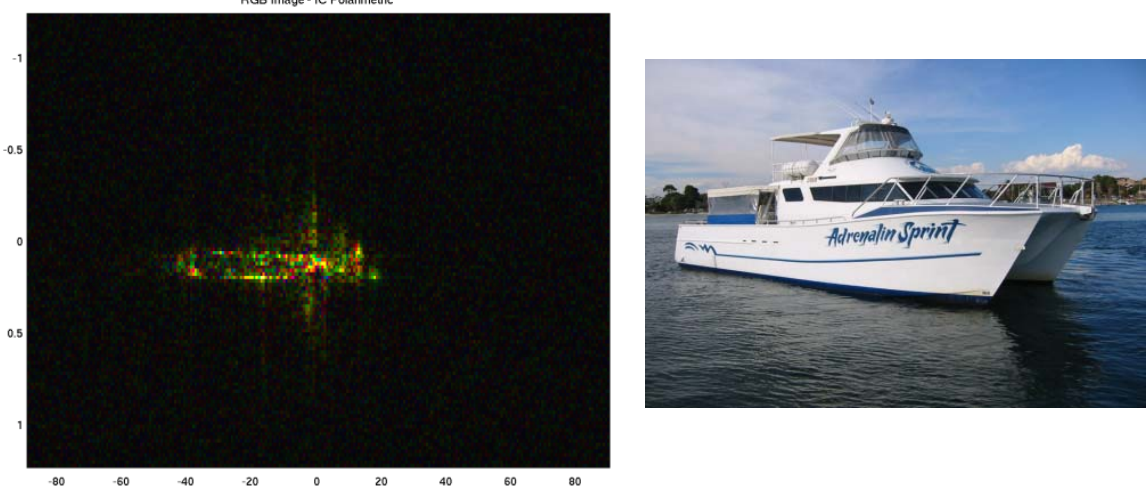

Figure 4: Polarimetric ISAR images. $\mathrm{R}=\mathrm{HH}+\mathrm{VV}, \mathrm{G}=\mathrm{HH}-\mathrm{VV}, \mathrm{B}=2 \mathrm{HV}$ 
Moreover, a 2D HF-OTH radar is also a very complex system because it is characterised by a set of features which are very unusual in comparison to ordinary microwave radars:

1. Transmission frequency must be selected upon the ionosphere propagation behaviour in the wide band [3-30 MHz].

2. Long radar coverage is allowed up to $4000 \mathrm{~km}$ corresponding to a zero antenna elevation angle. In practical applications it is convenient to limit the maximum distance to about $3000 \mathrm{~km}$ to avoid low antenna elevation angles as well as layer ionosphere internal multipath.

3. When the ionosphere EM incidence angle is greater than a critical value, the transmitted signal is not reflected and no returns occur. This phenomenon produces a blind area for distances less than about 400-600 km.

4. Ionosphere channel behaviour depends on date, sun activity and spatial coordinates. Therefore, ionosphere propagation changes widely depending on the time (night and day).

5. In the HF band, radar performance is heavily affected by background noise, which is mainly due to external noise. More precisely, the external noise is composed by atmospheric noise, cosmic noise and man-made noise. Internal noise caused by thermal effect is almost negligible.

6. We must deal with heavy propagation losses due to the long travelling distances as well as strong absorption losses caused by the ionosphere dispersion. The whole loss contribution can be up to 100-150 dB.

7. The apparently simple propagation mechanism hides the complexity of the ionosphere structure. This implies a challenging target localization that could be achieved by a smart system calibration combined with a three dimensional reconstruction of the signal path through the ionosphere.

8. OTH radar system functionalities strongly depend on the ionosphere and on the environment noise level that means performances is geographically dependent. Accordingly, the radar location represents one a key issue.

9. The principle of operation for a HF OTH sky-wave radar shows a spatial resolution cell that is range dependent.

10. The antenna system requirements are particularly demanding. It is remarkable that the radiating system should operate on a very wide frequency range (HF band).

11. High values of peak power are necessary in such systems to deal with strong losses. This requirement makes the antenna site more constrained in order to comply with national laws on EM radiation limits. 
12. HF radar cross section (RCS) of targets is regulated by different mechanism than in microwave regions. Targets lie in the Rayleigh and Mie region reporting a wide range of values. A simulative approach is essential in that it can provide a predicted RCS variability as a function of the operating frequency and of the aspect angles that are unusual for ordinary radar systems.

To have an idea of the complexity of such a system, Figure 5 shows the main blocks composing the architecture of an HF-OTH radar.

Although this radar is a very complex system, it could be a possible new sensor to be added to the existing systems to facilitate surveillance operations in a wide are such as the Mediterranean Sea.

\section{Satellite sensor constellations}

An earth observation satellite could be another interesting solution for wide area surveillance. These systems are equipped with different sensors like SAR, scatterometers, radar altimeters and IR and optical instruments. A wide field of view of such sensors allows to observe wide areas. The main drawback of such a system is the revisiting time related to the Low Earth Orbit (LEO) orbits, which is typically one or more days. Recently, constellations of satellites have been launched to reduce the revisiting time to a few hours. An example of such a system is the COSMO SKYMED

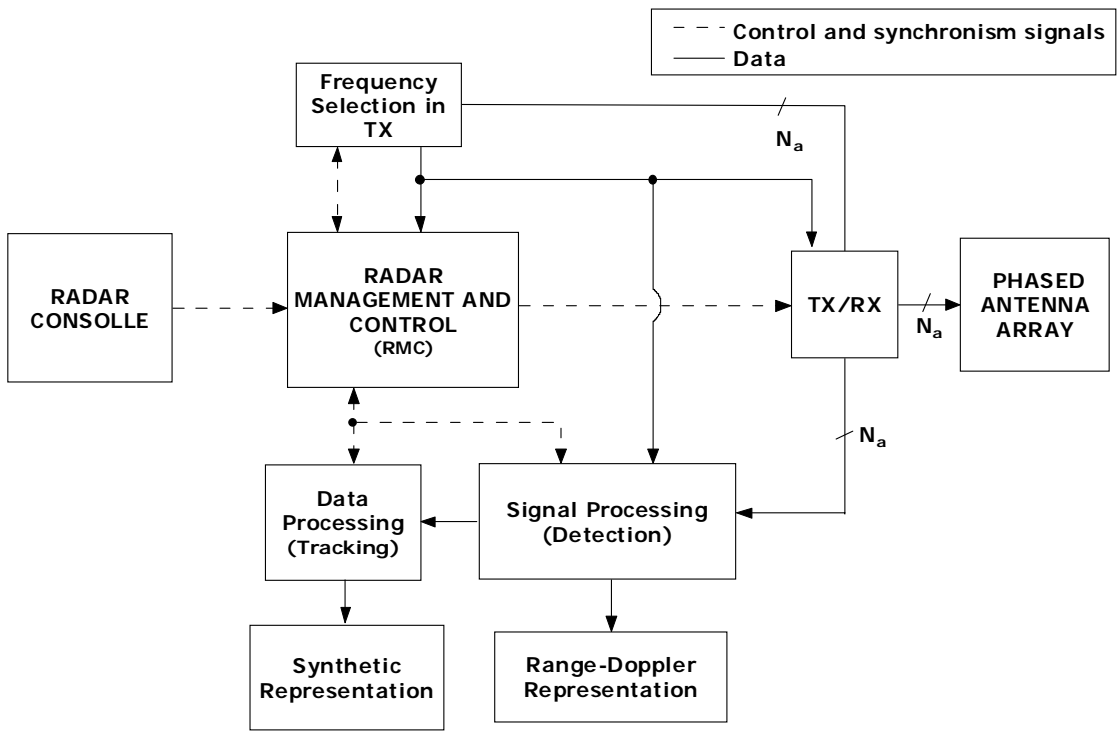

Figure 5: 2D HF radar architecture. 
constellation (see Figure 6). It consists of a constellation of four LEO mid-sized satellites, each equipped with a multi-mode high-resolution Synthetic Aperture Radar (SAR) operating at X-band and fitted with particularly flexible and innovative data acquisition and transmission equipment. Two satellites were launched in 2007. The full constellation was expected to be completely operational by 2010-2011. Different operating modes are possible (see Figure 7). Attainable spatial resolutions are reported in Figure 8. When the SAR operates in SCANSAR mode, wide swath up to $200 \mathrm{~km}$ can be obtained at the cost of spatial resolution that becomes $100 \times 100 \mathrm{~m}$. In STRIPMAP mode the spatial resolution improves to $5 \times 5 \mathrm{~m}$. The best performance is obtained in SPOTLIGHT mode where $1 \times 1 \mathrm{~m}$ resolution is achieved but reducing the coverage down to $10 \mathrm{~km}$. Anyway, the Cosmo Skymed missions are programmable to fit specific surveillance requirements coming from the MSA system. Revising time is short enough to monitor and track slow target like ships. For faster boats, just one shot image can be used.

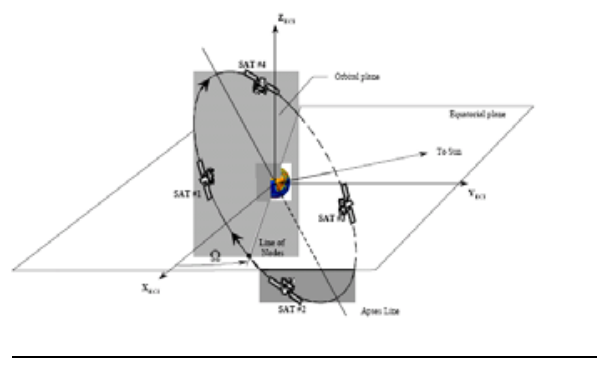

(a)

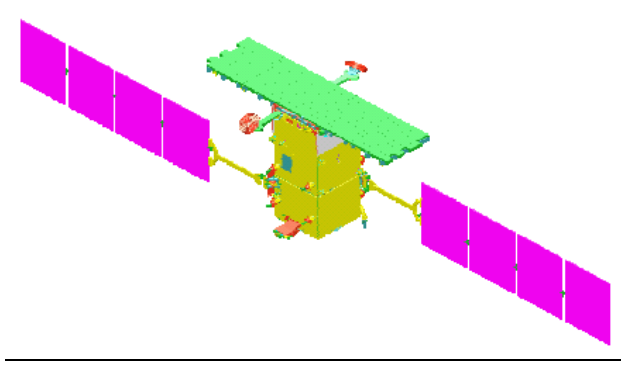

(b)

Figure 6: Cosmo Skymed Satellite orbit (a) and system (b).

We can state that such radars, suitably integrated with other sensors, could be another interesting innovative solution for improving MSA system performances.

\section{Considerations on Signal Processing and Data Fusion Techniques Advanced Signal processing techniques}

Several innovative and more efficient signal processing techniques have been recently proposed in the literature. In this sub-section we specifically refer to radar target detection.

Three different techniques for detection of targets from single or multiple polarization sea SAR images deserve a special attention. 


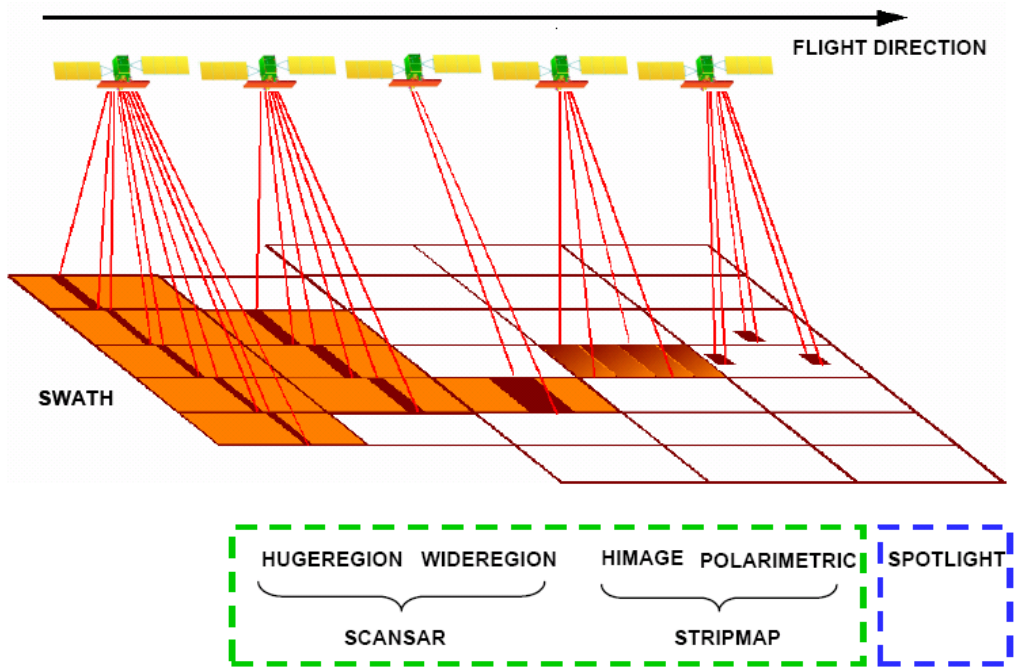

Figure 7: Cosmo Skymed operating modes.

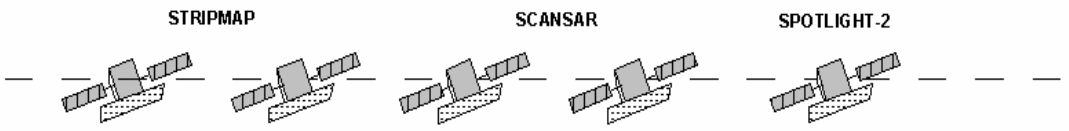

$619 \mathrm{~km}$

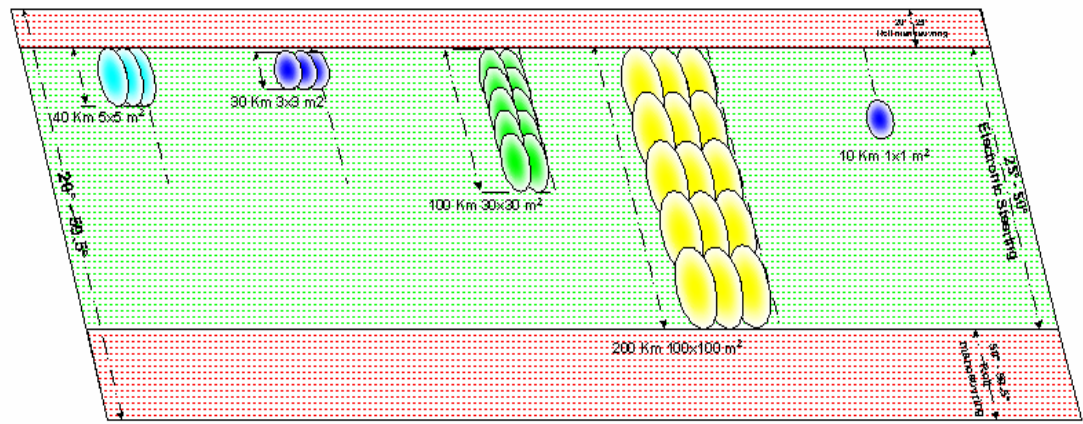

Figure 8: Cosmo Skymed SAR resolutions. 
- 2D-CFAR based technique;

- Fractal-based technique;

- $\quad$ STAP detection.

Adaptive 2D-CFAR techniques have been defined based on the amplitude distribution and correlation of the sea SAR clutter. A rectangular window moves along the SAR image, and the CFAR threshold of the Cell Under Test (CUT) at the centre of the window is computed by estimating the correlation matrix from the surrounding secondary pixels. In such a way, the detection threshold is spatially adaptive to guarantee Constant False Alarm Rate (CFAR) in the whole image.

Fractal morphological filtering based on Fractal FARIMA or FEXP models of the sea SAR images have been developed for improving Signal to Clutter. ${ }^{21,22,23}$ An example of ship detection from SAR images is reported in Figure 9. It is worth noting that the above techniques can be also applied to HRRPs and extended to target detection in ground clutter by suitably tuning the CFAR and morphological filter parameters.

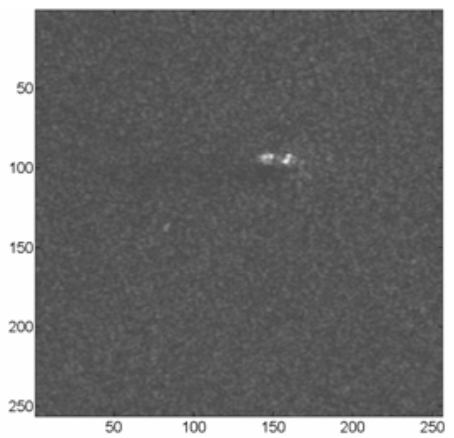

a)

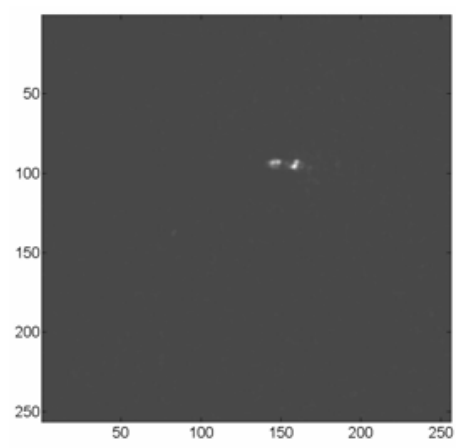

b)

Figure 9: Target detection from sea SAR image with fractal morphological filtering: a) before, and $b$ ) after filtering.

Another interesting technique is the Space Time Adaptive Processing (STAP). In array radars the antenna is composed of several radiant elements used both for reception and for transmission. Beamforming is typically used to determine the Direction of Arrival (DoA) of the target echo. Such a technique is often known as Space Adaptive Processing (SAP). Although the received signal is adaptively spatially filtered ground or sea clutter is still superimposed to the target echo thus reducing the Signal to Clutter Ratio (SCR). If Doppler processing were added to SAP, better SCRs could 
be obtained. This is the rationale of the STAP (Space Time Adaptive Processing). This technique is a sort of two-dimensional adaptive matched filtering of the received signal in the spatial-Doppler domain. STAP is typically applied to 1D or 2D Cartesian array (elements located in a uniform grid), but it is worth demonstrating that the STAP algorithm is also applicable to sparse arrays where positions of receiving elements are pseudo-random.

A few studies on the implementation of a 3D STAP (range, azimuth and elevation of detected target are estimated) through the use of a suitable combinations of a set of 2D STAPs (conical angle of DoA and range are estimated) has been recently studied at the University of Pisa. The main idea is to decompose a complex array in linear sub-arrays, use the 2D-STAP and combine the results to detect the target in a given range cell also providing the DOA and the Doppler frequency (radial velocity). Figure 10 shows the basic idea by referring to $2 \mathrm{D}$ orthogonal arrays.
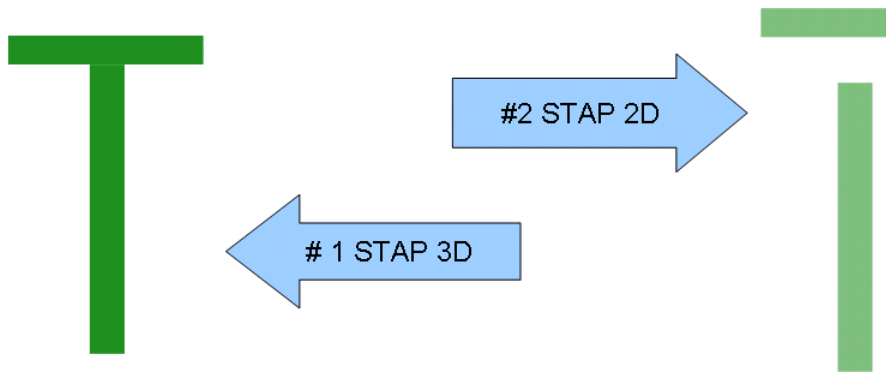

Figure 10: D-STAP as a combination of 2D STAPs.

Each 2D STAP provides a conical angle ambiguity of the echo DOA along its orthogonal direction (see Figure 11). The combined use of the two 2D STAP outputs allows a correct estimation of the target echo DOA (see Figure 12).

By using these new methods, the STAP technique is becoming feasible from a computational point of view also for a large array. The introduction of STAP in the new generation of array radar sensor could be another step toward MSA system improvement.

\section{Data fusion techniques}

Data fusion technologies involve the fusion of multi-sensory data to estimate the position, speed, attributes and identity entities. The low level processing techniques are relatively mature. Current research involves Multiple Hypothesis Trackers (MHT) 


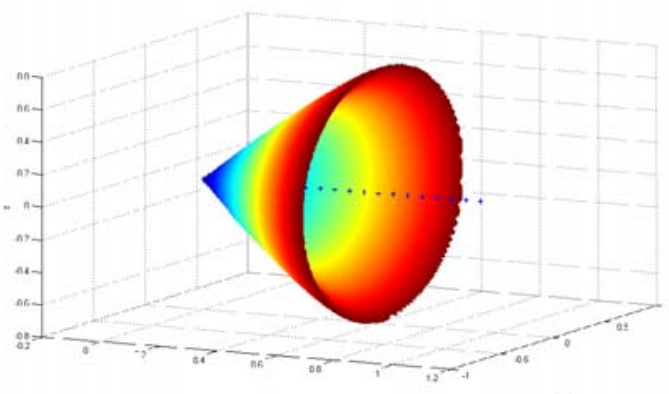

Figure 11: Conical angle ambiguity of a linear array 2D-STAP.

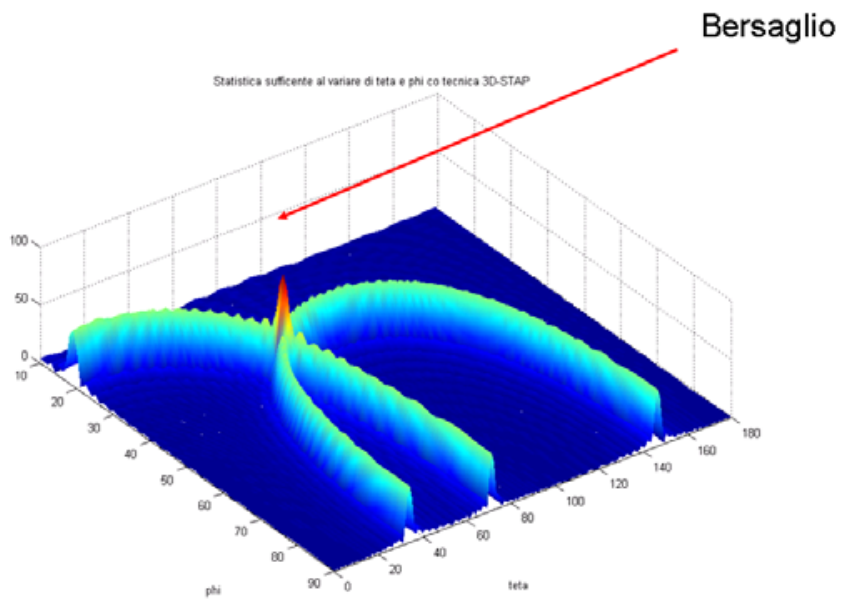

Figure 12: DOA estimation from 2D STAP conical angles.

and joint probabilistic data association (JPDA) trackers. Research addresses the use of simultaneous optimization approaches that seek to simultaneously estimate the state of multiple target and the assignments of observations to tracks. Tracking difficulties arise in dense target environments, low signal to noise observations, high clutter and noise and manoeuvring targets.

Feature based methods, mainly Artificial Neural Networks, are applied to determine target identity at feature level. An emerging trend is to apply hybrid methods for ro- 
bust target identification, like expert systems, rules and syntactic information about target composition, physics based models and patterns learned through neural networks. Identification at decision level is based on Bayesian inference, fuzzy logic, Dempster-Shafer's method and voting techniques. Such theories require understanding how to represent and propagate uncertainty. However, no unified concept of "uncertainty" exists that is shared by all these methods. The information fusion takes benefit from robust and flexible software packages for performing estimation and classification, and a methodology and guidelines to select algorithms.

Higher level data fusion analyses the results of low level processing to develop a context-based interpretation of the observed situation. This involves automatic reasoning to understand how observed entities interact with each other and the environment and to develop alternate hypotheses about future threat conditions.

The highest level of analysis is the meta-processing, which monitors the ongoing data fusion process to determine how to optimize the fusion results. This involves the control of sensors, providing dynamic adjustments to the fusion algorithms, and monitoring the quality of the fusion. Actually the combined usage of different sensors is not standardized and using geographically distributed sensors to observe physically different phenomena at different rates of time is particularly difficult. In addition, very little research has been performed to link the human decision maker with sensor tasking and control of fusion algorithms. Problems include the lack of measures of effectiveness to quantify the performance of the fusion, a gap between the optimization of a mission and the real time control of sensors and algorithms, and difficulties in dealing with multi platform systems.

At the state-of-the-art of content-based retrieval, most results emanate from the field of image retrieval, video-based retrieval still being in its infancy. Experimental systems like Simplicity, ${ }^{24}$ Amico, ${ }^{25}$ IBM QBIC, ${ }^{26}$ Columbia VideoQ,${ }^{27,28}$ exist, but they all have their deficiencies. Many systems are based on textual search, ${ }^{29,30}$ which require manual annotation of the contents. Content-based experimental systems usually work by comparing colour histograms, combined colour and texture features, DCT or wavelet coefficients, and/or the special arrangement of such features. There are problems not only with the algorithms or their speed, but with their use cases too: users might wish to search for similar images, for some class of similar images, for some class of images with a particular theme, just browse over a set of images, combine metadata and content queries, etc. Most systems cannot handle more than one or two of these search criteria, and they cannot handle content-based video searches reliably (if at all). Fusion of video information to other sensors is a new improvement. The cross-modal evaluation of alarming signals or the multimodal definition of Region-of Interest (ROI) areas in a confuse situation is a great challenge where important developments may be forthcoming. 
Work on object recognition falls into two broad categories: recognition of individual objects and recognition of categories. Single objects are easier to manage; hence, advanced methods have been designed for efficient recognition, lighting and viewpoint invariant ${ }^{31}$ representation and recognition. Recognition of categories is more general; therefore it requires complex models which are difficult to learn. Most of the work in this field has been concentrated on the modelling of objects and the learning of model parameters. Existing recognition methods model either the geometrical relationships between certain features, ${ }^{31}$ or model the appearance of objects. ${ }^{32,33}$ In the past, several methods have been developed which exploit single or multiple view dynamic information for object detection or view matching. ${ }^{34,35}$

Visual surveillance is important in location, tracking and tracing. Several studies use pixel based analysis (empirical, Mixture of Gaussians). ${ }^{36}$ Other use Hidden Markov models (HMM) to learn the temporal behaviour of moving objects, however most of these methods are based on tracking ${ }^{37}$ and will fail where large numbers of objects are moving simultaneously. Instead low-level features (optical flow, localized motion direction) can be used in MOGHMM (Mixture of Gaussians HMM) to learn the fluctuation of the traffic, and unusual events are indicated by the sudden change in the fluctuation. ${ }^{38,39}$

The management of the data in support of data fusion is actually based on commercial Data Base Management Systems (DBMS), which use relational data models and query languages to provide access to data. Recently, natural language interfaces are being developed along with object oriented databases. Traditionally, databases address one of the following types of data: text and numbers, graphic, symbolic information (e.g. rules and ontologies); there is no a single DBMS which attempts to handle all the three classes of data. A problem of DBMS in data fusion is the inability to simultaneously optimize rapid storing of sensor data and the data retrieval required by users and fusion algorithms. Another challenge is how to ensure synchronization among distributed databases. Finally, DBMS should improve their security at different levels, ensuring that authorized persons can access the data and processing results.

\section{Conclusion}

A Maritime Situational Awareness system concept for Mediterranean sea surveillance has been presented. A general classification of Maritime Surveillance Systems (MSS) has been presented and main potentials and shortfalls of existing MSSs operating on the Mediterranean Sea were analysed and compared (with a highlight on the Italian MSS structure). Solutions to enhance technology gaps were suggested. MSA system architecture, applications, requirements (focusing on technical, legal and administrative features) and sensors were proposed with a few considerations on signal proc- 
essing and data fusion techniques, required for the implementation of the MSA system capabilities.

This MSA concept attempts to foresee an integrating surveillance system at EU level focusing on the Mediterranean countries. Achieving a MSA system of systems concept needs to improve and integrate the existing technologies (communications, sensors, etc.) but on the other hand needs to increase the effectiveness of maritime surveillance authorities by making available more tools and more information necessary for the performance of their duties. Cooperation at EU level is the core of any surveillance integrated architecture and joint based initiatives and projects would be the best cost/effectiveness solutions.

\section{References:}

1. M. Martorella, et al., "Time Windowing for highly focused ISAR Image Reconstruction," IEEE Trans. on Aerospace and Electronic System 41:3 (July 2005): 992-1007.

2. M. Martorella, et al., "A Contrast Maximization Based Technique for 2D ISAR Autofocusing," IEE Proceeding on Radar Sonar and Navigation 152:4 (August 2005): 253262.

3. M. Martorella, et al., "Use of Genetic Algorithms for Contrast Maximization and Entropy Minimization in ISAR Autofocusing," EURASIP Journal on Applied Signal Processing (April 2006), Article ID 087298, pp. 1-11, DOI 10.1155/ASP/2006/87298.

4. M. Martorella, et al., "Inverse Synthetic Aperture Radar," EURASIP Journal on Applied Signal Processing (April 2006), Article ID 63465, pp. 1-4, DOI 10.1155/ASP/2006/63465.

5. M. Martorella, et al., "Statistical CLEAN Technique for ISAR Imaging," IEEE Tr. on Geoscience and Remote Sensing 45:11 (November 2007): 3552-60.

6. F. Berizzi, et al., "A Range Profiling Technique for Synthetic Wideband Radar," Proceedings of IET-RSN 2:5 (October 2008): 334-50.

7. E. Giusti, et al., "Equivalence between Cameron's Unit Disc and Poincaré's Sphere for Symmetric Scattering Characterisation and Classification," IEEE Geoscience and Remote Sensing Letters 5:2 (April 2008): 152-56.

8. F. Berizzi, et al., "A Contrast Based Algorithm for Synthetic Range Profile Motion Compensation," IEEE Tr. On Geoscience and Remote Sensing 46:10 (October 2008): 305362.

9. M. Martorella, et al., "Polarimetric ISAR Autofocusing," IET Signal Processing 2:3 (September 2008): 312-24.

10. M. Martorella, et al., "CLEAN Technique for Polarimetric ISAR," International Journal of Navigation and Observation - Special issue on "Modelling and Processing of Radar Signals for Earth Observation" (2008), ID 325729, 12 pages. 
11. M. Martorella, R. Soleti, F. Berizzi, E. Dalle Mese "Plume Effect on Radar Cross Section of missiles at HF band," in Proceeding of the International Radar Conference, 3-5 September 2003, Adelaide (Australia).

12. M. Martorella, B. Haywood, F. Berizzi, E. Dalle Mese "Performance Analysis of an ISAR Contrast Based Autofocusing Algorithm Using Real Data," in Proceeding of the International Radar Conference, 3-5 September 2003, Adelaide (Australia).

13. F. Berizzi , E.Dalle Mese, A. Monorchio, Brizzi, L. Rainone, "Radar Cross Section of Military Targets at HF Band," in Proceeding of "Military Sensing" conference, October 2004, Dresden (Germany), IT_113.

14. F.Berizzi, A.Capria, E. Dalle Mese, R.Soleti, "A Method for Peak Power Evaluation for HF-OTH Skywave Radar," in Proceedings of the 2006 International Radar Symposium (IRS 2006), Kracòw (Poland), May 2006.

15. F. Berizzi, , R. Soleti, A. Capria, E. Dalle Mese, "A frequency selection method for HFOTH skywave radar systems," in Proceeding of EUSIPCO'06 conference, Florence (IT), September 2006.

16. F. Berizzi, E. Dalle Mese, A. Monorchio, A. Capria, R. Soleti, "HF Radar Sensors for Theatre Surveillance," IV Simposio sulle Tecnologie Avanzate, Roma, Italy, June 2007.

17. F. Berizzi, R. Soleti, A. Capria, E. Dalle Mese, I. Gulino, "A Simulation Framework for HF-OTH Skywave Radars: The Received Signal Model," in Proceeding of International Radar Symposium (IRS’07), Cologne (Germany), September 2007.

18. F. Berizzi, E. Dalle Mese, A. Capria, R. Soleti, "HF-OTH Skywave Radar for Missile Detection," Symposium on "Sensors and Technology for Defence Against Terrorism," 22-25 April 2008, Mannheim, Germany.

19. S.J.Anderson, F. Berizzi, C. Bianchi, "Generation and Assimilation of Propagation Advice for HF Skywave Radar Systems,” IEEE Radar Conference, Rome, 26-29 May 2008.

20. F. Berizzi, E. Dalle Mese, A. Monorchio, A. Capria, R. Soleti, "On the Design of a 2D Array HF Skywave Radar,” IEEE Radar Conference, Rome, 26-29 May 2008.

21. M. Bertacca, F. Berizzi, E. Dalle Mesep "A FARIMA Based Technique for Oil Slick and Low Wind Areas Discrimination in Sea SAR Imageryp" IEEE Trans. on Geoscience and Remote Sensing 43:11 (November 2005): 2484-93.

22. F. Berizzi, M. Bertacca, E. Dalle Mese, "FEXP Models for Oil Slick and Low-Wind Areas Analysis and Discrimination in Sea SAR Images," in Proceedings of the SeaSAR 2006 Workshop, ESA-Esrin, Frascati, Roma, 23-26 January 2006.

23. F. Berizzi, M. Bertacca, E. Dalle Mese, "Homomorphic Inverse-FEXP Filtering for Target Detection in Sea SAR Images," in Proceedings of the 2006 International Radar Symposium (IRS 2006), Kracòw (Poland), May 2006.

24. SIMPLICITY, http://wang14.ist.psu.edu/cgi-bin/zwang/regionsearch_show.cgi at Stanford, Semantics-sensitive Integrated Matching for Picture LIbraries.

25. The Art Museum Image Consortium (AMICO), http://www.amico.org/.

26. IBM's Query by Image Content, IBM QBIC, http://wwwqbic.almaden.ibm.com/.

27. Columbia VideoQ, http://www.ctr.columbia.edu/VideoQ.

28. G. Carneiro, A.B. Chan, P.J. Moreno, N. Vasconcelos, "Supervised Learning of Semantic Classes for Image Annotation and Retrieval," IEEE Transactions on Pattern Analysis and Machine Intelligence 29:3 (2007): 394-410.

29. Google video search, http://video.google.com/. 
30. Yahoo video search, http://video.search.yahoo.com/.

31. W. Zhao, R. Chellappa, R. Rosenfeld, "Face recognition: A Literature Survey," ACM Computing Surveys (2003): 399-458.

32. P. Viola, M. J. Jones, "Robust Real-Time Face Detection," International Journal of Computer Vision 57:2 (2004): 137-54.

33. L. Fei-Fei, R. Fergus and P. Perona, "One-shot learning of object categories," IEEE Transactions on Pattern Analysis and Machine Intelligence 28 (2006): 594-611.

34. Z. Szlávik, T. Szirányi, L. Havasi, "Stochastic View Registration of Overlapping Cameras Based on Arbitrary Motion," IEEE Transactions on Image Processing 16:3 (2007): 71020.

35. L. Havasi, Z. Szlávik, T. Szirányi, "Detection of Gait Characteristics for Scene Registration in Video Surveillance System," IEEE Transactions on Image Processing 16:2 (2007): 503-10.

36. Á. Utasi, L. Czúni, "Anomaly Detection with Low-level Processes in Videos," The 3rd International Conference on Computer Vision Theory and Applications, Funchal, Portugal, 22-25 January 2008.

37. V. Nair, J.J. Clark, "Automated Visual Surveillance Using Hidden Markov Models," in Proceedings of the 15th Vision Interface Conference (2002), pp. 88-92.

38. M. Brand and V. Kettnaker, "Discovery and segmentation of activities in video," IEEE Trans. Pattern Analysis and Machine Intelligence 22:8 (August 2000): 844-51.

39. Á. Utasi, L. Czúni, "HMM-based Unusual Motion Detection without Tracking," The 19th International Conference on Pattern Recognition, Tampa, USA, 8-11 December 2008.

40. P. Sen and A. Deshpande, "Representing and Querying Correlated Tuples in Probabilistic Databases," IEEE 23rd International Conference on Data Engineering (2007), pp. 596605.

41. European Commission, Non-paper on Maritime Surveillance, 2008.

Fabrizio BERIZZI, Amerigo CAPRIA, Enzo DALLE MESE, and Marco MARTORELLA are with the Department of "Ingegneria dell'Informazione" at the University of Pisa, Via Caruso 16, 56122 Pisa, Italy. E-mail of the corresponding author: f.berizzi@iet.unipi.it.

Amleto GABELLONE is with the Naval Institute for Electronics and Communications "G. Vallauri", Viale Italia 72, 57126 Livorno, Italy. E-mail: amleto.gabellone@marina.difesa.it. For Mr. Gabellone’s CV see p. 43 of this volume.

Walter MATTA is with Vitrociset S.p.A., Via Salaria 102700138 Roma, Italy.

E-mail:w.matta@vitrociset.it. 